\title{
Zum Inhalt von Band IV
}

Der vorliegende vierte Band „Optik, Strahlung“ zeigt zunächst, dass kohärente Wellen interferieren. Dies führt zu Beugungserscheinungen an Begrenzungen des Strahlungsfeldes. Zwischen dem Strahlungsfeld am beugenden Hindernis und der Beobachtung der Intensitätsverteilung vermittelt die Fouriertransformation. Es folgt die Beschreibung von Reflexion und Transmission mit den Fresnelschen Formeln sowie die Besprechung der experimentell wichtigen Spektralapparate und Interferometer. In der geometrischen Optik werden Beugungserscheinungen vernachlässigt und die geradlinige Lichtausbreitung zugrunde gelegt. Spiegel, Prismen und vor allem Linsen sind die Basis für optische Instrumente. Mit dem Kapitel „Wärmestrahlung“ wird in diesem Band zum ersten Mal die Grenze von der klassischen Physik zur Quantenphysik überschritten: Die Vorstellung, dass sich die Strahlungsenergie, die ein heißer Körper abgibt oder aufnimmt kontinuierlich verändern kann, muss aufgegeben werden. Die Plancksche Quantenhypothese führt auf das Plancksche Strahlungsgesetz, das die Intensitätsverteilung der Strahlung des „schwarzen Körpers“ richtig beschreibt. 
